\title{
Sexually dimorphic venom proteins in long-jawed orb-weaving spiders (Tetragnatha) comprise novel gene families
}

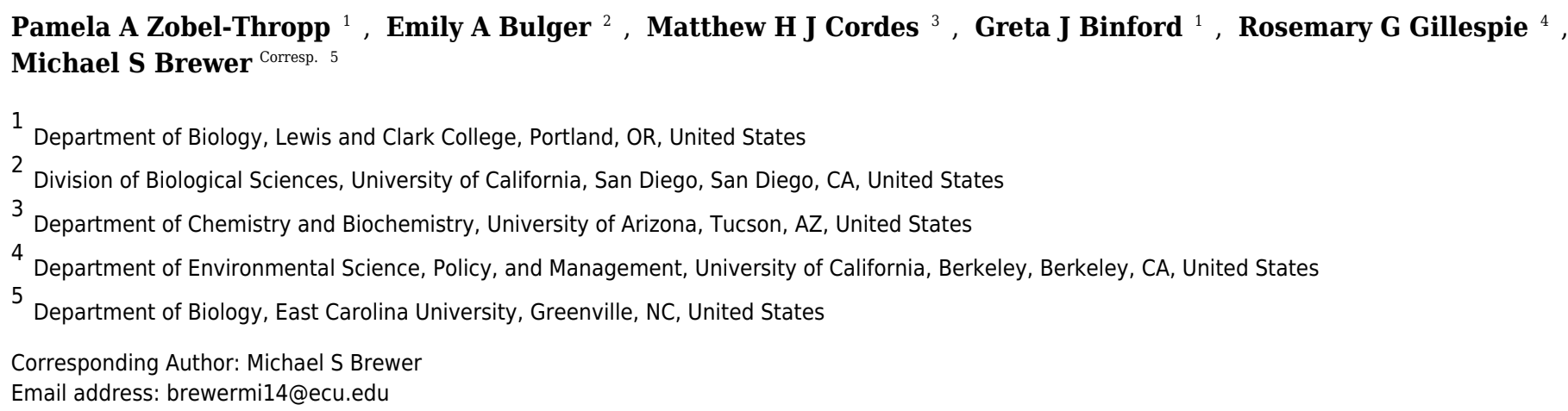

Venom has been associated with the ecological success of many groups of organisms, most notably reptiles, gastropods, and arachnids. In some cases, diversification has been directly linked to tailoring of venoms for dietary specialization. Spiders in particular are known for their diverse venoms and wide range of predatory behaviors, although there is much to learn about scales of variation in venom composition and function. The current study focuses on venom characteristics in different sexes within a species of spider. We chose the genus Tetragnatha (Tetragnathidae) because of its unusual courtship behavior involving interlocking of the venom delivering chelicerae (i.e., the jaws), and several species in the genus are already known to have sexually dimorphic venoms. Here, we use transcriptome and proteome analyses to identify venom components that are dimorphic in Tetragnatha versicolor. We present cDNA sequences including unique, male-specific high molecular weight proteins that have remote, if any, detectable similarity to known venom components in spiders or other venomous lineages and have no detectable homologs in existing databases. While the function of these proteins is not known, their presence in association with the cheliceral locking mechanism during mating together with the presence of prolonged male-male mating attempts in a related, cheliceral-locking species (Doryonychus raptor) lacking the dimorphism suggests potential for a role in sexual communication. 
4 Sexually dimorphic venom proteins in long-jawed orb-weaving spiders (Tetragnatha) 5 comprise novel gene families

6

7 Pamela A. Zobel-Thropp ${ }^{1}$, Emily A. Bulger ${ }^{2}$, Mathew H.J. Cordes ${ }^{3}$, Greta J. Binford ${ }^{1}$, Rosemary

8 G. Gillespie ${ }^{4}$, Michael S. Brewer ${ }^{5}$

9

${ }^{1}$ Department of Biology, Lewis and Clark College, Portland, OR, USA

11 2Division of Biological Sciences, University of California, San Diego, San Diego, CA, USA

$12{ }^{3}$ Department of Chemistry and Biochemistry, University of Arizona, Tucson, AZ, USA

$13{ }^{4}$ Department of Environmental Science, Policy, and Management, University of California,

14 Berkeley, Berkeley, CA, USA

$15{ }^{5}$ Department of Biology, East Carolina University, Greenville, NC, USA

16

17 Corresponding Author:

18 Michael S. Brewer ${ }^{5}$

19

20 Email address: brewermi14@ecu.edu

21

22

23 


\section{ABSTRACT}

25 Venom has been associated with the ecological success of many groups of organisms, most notably 26 reptiles, gastropods, and arachnids. In some cases, diversification has been directly linked to 27 tailoring of venoms for dietary specialization. Spiders in particular are known for their diverse 28 venoms and wide range of predatory behaviors, although there is much to learn about scales of variation in venom composition and function. The current study focuses on venom characteristics in different sexes within a species of spider. We chose the genus Tetragnatha (Tetragnathidae) because of its unusual courtship behavior involving interlocking of the venom delivering chelicerae (i.e., the jaws), and several species in the genus are already known to have sexually dimorphic venoms. Here, we use transcriptome and proteome analyses to identify venom components that are dimorphic in Tetragnatha versicolor. We present cDNA sequences including unique, male-specific high molecular weight proteins that have remote, if any, detectable similarity to known venom components in spiders or other venomous lineages and have no detectable homologs in existing databases. While the function of these proteins is not known, their presence in association with the cheliceral locking mechanism during mating together with the presence of prolonged male-male mating attempts in a related, cheliceral-locking species (Doryonychus raptor) lacking the dimorphism suggests potential for a role in sexual communication.

\section{INTRODUCTION}

43 Phenotypic differences between sexes are widespread among animals and can be attributed to 44 sexual and/or natural selection. Sexual selection is the most common explanation for morphological and behavioral differences, which are frequently attributed to intra- or intersexual 
47 bimodal or dimorphic niche separation between the sexes (Berns, 2013). Recent work has

48 highlighted the importance of additional modalities that differ between sexes, including acoustic

49 (Elias \& Mason, 2014) and chemical (Wyatt, 2014b) traits. Here we focus on sexual differences

50 in chemical traits, and more specifically on venom, in which several studies have highlighted

51 variation according to sex. We examine sexual differences in venom composition of spiders, and

52 consider mechanisms of selection that may have given rise to these differences.

Venoms are complex chemical cocktails that attract research attention in both applied and

54 basic sciences and have been characterized in many animals, including mammals (shrews, 55 platypus), toxicoferan reptiles (lizards and snakes), fish, sea anemones, cephalopods, cone snails,

56 insects, centipedes, scorpions, shrews, and arachnids (Fry et al., 2009). They can be impressively 57 complex; among spiders in particular, individual venoms can have 1000s of components 58 (Escoubas, 2006). The complexity typically consists of related sets of molecules within which are 59 components with exquisite functional specificity, and novel activities (reviews in (Kuhn-Nentwig 60 \& Stöcklin, 2011; Smith et al., 2013; King, 2015). Activities involve manipulation of physiological

61 processes, particularly neurological. Thus they are a source for discovery of components with 62 human applications in pharmaceuticals or insecticides (King, 2015).

Venoms frequently exhibit sexual dimorphism (snakes, (Menezes et al., 2006); scorpions,

64 (D'Suze, Sandoval \& Sevcik, 2015; Miller et al., 2016); spiders, (Herzig et al., 2008; Binford, 65 Gillespie \& Maddison, 2016)). Because the primary functional roles of venoms in spiders are 66 thought to be predation and defense, in most cases sexual differences in venom composition 67 between adults of many species are hypothesized to result from natural selection optimizing 68 composition based on differences in feeding biology and associated differences in diet composition 69 and/or vulnerability to predation. Thus, hypotheses to explain sexual dimorphism in spider venoms 
70 typically center on differential optimization of chemical pools for divergent adult niches. Though

71 largely untested, these hypotheses provide plausible explanations for many known dimorphisms.

72 For example, in Sydney funnel-web spiders (Atrax robustus), male venoms are more toxic to

73 mammals than are those of females (Gray \& Sutherland, 1978), potentially associated with male

74 Atrax being found more often wandering outside of burrows than females (Isbister \& Gray, 2004).

75 In contrast, in the theridiid spiders Latrodectus mactans and Steatoda paykulliana, female venoms

76 have higher mammalian neurotoxic activity than male venoms (Maretić, Levi \& Levi, 1964);

77 however, as the volumes of venom were not controlled, observed differences may be due to the

78 greater amount of venom injected by the larger females. These spiders eat vertebrates and bite

79 humans defensively, so the general pattern of increased female potency has been attributed to the

80 shorter lifespan of male spiders and reduced adult foraging of males (Rash, King \& Hodgson, $812000)$.

82 The long-jawed orb-weaving spiders (Araneae: Tetragnathidae: Tetragnatha) provide a 83 compelling context to explore the nature and potential cause of sexual dimorphism in venoms.

84 Members of the genus Tetragnatha are broadly distributed with $c a .347$ species worldwide (World 85 Spider Catalog version 19, 2018). The majority of species worldwide are remarkably uniform in 86 appearance, dull brown or olive in color, with long first and second legs, typically long jaws in 87 adulthood, and an elongate opisthosoma (Levi, 1981). Their behavior and ecology is also fairly 88 homogeneous as they generally construct a light and fragile orb web with an open center and build 89 the web over water or in other wet places (Gillespie, 1987), although they have undergone adaptive 90 radiation in the Hawaiian Islands, associated with marked shifts in ecology and behavior 91 (Gillespie, 2004; Blackledge \& Gillespie, 2004). 
92 Comparisons of crude venoms between sexes of Tetragnatha using 1-D protein

93 electrophoresis have identified a particularly striking sexual dimorphism in which males have an

94 abundance of high molecular weight components that are not present in females (Binford, Gillespie

$95 \&$ Maddison, 2016). Phylogenetic comparisons across species indicate that these high molecular

96 weight components persist across an evolutionary transition in feeding biology that reduces the

97 differences in adult feeding niches. Specifically, adult males of orb-weaving Tetragnatha species

98 do not typically build webs and are functionally wandering predators. However, a lineage of

99 Tetragnatha in Hawaii has lost orb-weaving behavior (Gillespie, 2004; 2005) and both males and

100 females wander in search of prey, thus reducing dimorphism in feeding biology (Gillespie, 1991).

101 Males could be more prone to predation and have unique components that function in defense, but

102 if so, increased vulnerability would also affect female wandering Tetragnatha. Therefore, a 103 defensive role does not seem likely.

104 The goal of this study is to identify the molecules that are sexually dimorphic in venoms 105 of a readily accessible "model" species, Tetragnatha versicolor. Using comparative venom gland 106 transcriptomes and proteomes of adult males and females, we identify sequence characteristics of 107 dimorphic components, with particular attention to those unique to males. We infer function 108 preliminarily using homology searching and report the discovery of divergent highly expressed 109 male-specific proteins.

\section{MATERIAL AND METHODS}

112 Collection. Individuals of Tetragnatha versicolor were collected by hand from three populations:

113 along the southern fork of Strawberry Creek on the UC Berkeley Campus (UCB) (37.872 ${ }^{\circ}$, 
$\left.114122.262^{\circ} \mathrm{W}\right)$, Little Sugar Creek, Binford Farm (BF), Crawfordsville, Indiana (40.061 ${ }^{\circ} \mathrm{N}$,

$\left.11586.853^{\circ} \mathrm{W}\right)$, and Greenville, North Carolina (ECU) $\left(35.626^{\circ} \mathrm{N}, 77.409^{\circ} \mathrm{W}\right)$.

116 Venom Extraction. Live specimens were transported to Lewis \& Clark College where venom was

117 extracted by electrostimulation (Binford, 2001). We obtained venom samples from 19 females and

11816 males from UCB, nine females and two males from BF, and nine females and five males from

119 ECU. To compile sufficient protein amounts for proteomics analyses, venom was pooled within 120 sexes for each of these three populations.

$121 \boldsymbol{R N A}$ Isolation Trancsriptomic analyses were performed using RNA isolated only from Indiana

122 (BF) specimens. To capture some breadth of transcriptional timing after emptying venom glands,

123 surviving spiders (10 females and two males) were divided into two groups within each sex, and

124 venom glands were isolated from five females and one male two and three days after venom

125 extraction. The glands extracted two and three days after milking were pooled within sexes and

126 processed and analyzed separately between sexes for all subsequent analyses. To extract glands,

127 spiders were anesthetized with $\mathrm{CO}_{2}$ and venom glands were removed by dissection and flash-

128 frozen immediately in liquid nitrogen. Total RNA was isolated by grinding tissues in TRIzol®

129 reagent (Life Technologies, Carlsbad, CA), adding chloroform (200 $\mu \mathrm{L}$ per mL of TRIzol®),

130 mixing by inversion, and incubating for $20 \mathrm{~min}$ at $4^{\circ} \mathrm{C}$. The tube was centrifuged at $4^{\circ} \mathrm{C}$ for 15

$131 \mathrm{~min}$ at $14,000 \mathrm{rpm}$. An equal volume of cold $100 \%$ ethanol was added to the RNA-containing

132 upper aqueous phase. The solution was then passed through an RNeasy® Mini Spin Column

133 (Qiagen, Chatsworth, CA) and purified, according to RNeasy® protocols.

134 Illumina RNA Sequencing and Quality Control. RNA extractions from the BF population of $T$.

135 versicolor males (2) and females (10) were shipped to the Genomic Services Lab at HudsonAlpha

136 (Huntsville, AL), where cDNA libraries were prepared from total RNA (poly-A isolation, Illumina 
137 TruSeq RNA Library Prep Kit v2), and 50 bp paired-end Illumina HiSeq 2500 RNA-seq was used

138 to generate sequence reads. All QC trimming and assemblies were done using a pipeline provided

139 by the UC Berkeley Museum of Vertebrate Zoology (https://github.com/MVZSEQ). Quality and

140 GC content of the resulting paired-end reads was assessed using the FastQC v0.10.0 program

141 (http://www.bioinformatics.babraham.ac.uk/projects/fastqc/). TRIMMOMATIC v0.36 (Bolger,

142 Lohse \& Usadel, 2014) and CUTADAPT v1.7.1 (Martin, 2011) were used to clean up the sequence

143 data: nucleotides below a quality threshold of 20 were trimmed from the ends of sequences, and

144 sequences shorter than 36 nucleotides (after trimming) were discarded. The reads were aligned to

145 a custom library of bacterial sequences to remove prokaryotic contamination using BOWTIE2

146 v2.1.0 (Langmead \& Salzberg, 2012). Individual paired-end files were resynchronized, removing

147 any paired-end sequences only present in one of the two files. Left and right reads that overlap

148 were combined into a single longer read to aid in downstream assembly using FLASH v1.2.7

149 (Magoč \& Salzberg, 2011).

150 Transcriptome Assembly and ORF Prediction. The resulting male and female files were

151 assembled separately, by sex, and combined for a general T. versicolor venom transcriptome. The

152 processed reads for each sex and the combined read files were assembled using the TRINITY

153 pipeline v2.0.6 (http://trinityrnaseq.sourceforge.net/) with default parameters except the following,

154 group_pairs_distance=999 and min_kmer_cov=2. High-confidence open reading frames (ORFs)

155 (i.e., likely coding sequences), were obtained for each gene in the transcriptome using

156 TRANSDECODER r20140704 (Haas et al., 2013). A minimum protein length of 30 amino acids

157 was used in ORF predictions. The completeness of each assembly was assessed via BUSCO v1.1

158 (Simão et al., 2015). 
159 Read mapping to identify transcriptome dimorphisms. The processed reads from each sex were

160 mapped against the combined assembly to identify genes that are unique to either sex, with

161 particular emphasis placed on male-only transcripts. The mapping was performed using STAR

162 v2.4.2a (Dobin et al., 2012) and default parameters. A custom python script was used to generate

163 a BED file from the combined transcriptome and BEDTOOLS v2.18.1 "multicov" (Quinlan \&

164 Hall, 2010) was used to generate counts of reads from each sex mapping to combined assembly

165 transcripts. An additional round of mapping with BOWTIE v1.1.1 (Langmead et al., 2009)

166 followed by GFOLD v1.1.4 (Feng et al., 2012) analysis identified differentially expressed

167 transcripts between males and females (GFOLD cutoff $=2$, which approximates a $\log _{2}$ fold change

168 of two).

169 Functional annotation and GO enrichment analyses. The male, female, and combined 170 assemblies were annotated via the TRINOTATE pipeline (Haas et al., 2013). This approach 171 comprises the following steps. All contigs (BLASTX) and predicted amino acid sequences 172 (BLASTP) were searched against the Swissprot database (downloaded 23-iv-2015). Protein 173 domains were identified by running a HMMER v3.1b2 (Finn, Clements \& Eddy, 2011) search 174 against the PFAM ((Bateman et al., 2004); downloaded 23-iv-2015) database, and signal peptides, 175 indicating secreted proteins, were discovered with SignalP v4.1 176 (http://www.cbs.dtu.dk/services/SignalP/). TMHMM v2.0c (Krogh et al., 2001) was used to 177 annotate transmembrane domains. Finally, RNAMMER v1.2 (Lagesen et al., 2007) identified 178 rRNA transcripts. Results from database searches were loaded into a sqlite database, and GO terms 179 were applied and used in downstream analyses. GO enrichment analyses were performed on the combined assembly using the subset of 181 genes endemic to male spiders, as verified by the proteomic analyses outlined below. Two data 
182 sets were analyzed, 1) all male specific proteins and 2) high molecular weight ( $>43 \mathrm{kDa})$ male

183 proteins. This was done using scripts provided with the TRINITY and TRINOTATE software and

184 the R Bioconductor package “GOseq" v1.18.0 (Young et al., 2010).

185 Gene family reconstruction via Markov clustering. Predicted ORFs from the male, female, and 186 combined assemblies were combined into a single FASTA file, a BLAST database was created, 187 and the sequences were searched (BLASTP; e-value cutoff $=\mathrm{e} 10^{-5}$ ) against themselves (i.e., an 188 ALLvsALL BLAST). The results were clustered into putative gene families using the Markov 189 Clustering Algorithm (MCL v14.137; (Enright, Van Dongen \& Ouzounis, 2002)) with default 190 parameters and an inflation value of 2.0. The resulting e-values were negative log transformed, 191 and the results were separated using a heuristically chosen cutoff (-1.91) and results were 192 visualized in CYTOSCAPE v3.0.1 (Shannon et al., 2003). Clusters representing putative gene 193 families were used in subsequent analyses. Initial clustering identified a single cluster of particular 194 interest, cluster six, comprising high molecular weight proteins that was subsequently subclustered 195 (negative log transformed and heuristically chosen edge weight cutoff $=-0.93$ ).

196 Proteomic analyses. Crude venoms were dissolved in a standard buffer $(5 \mathrm{mM} \mathrm{CaCl} 2 / 50 \mathrm{mM}$ Tris, $197 \mathrm{pH}$ 8), pooled by sex, and shipped to the Arizona Proteomics Consortium. Venom-expressed 198 proteins were separated by size using SDS-PAGE (12\%, Fig 1C). To increase resolution, each lane 199 was divided into three sections and digested with trypsin followed by a clean-up step using C18 200 ZipTips (Millipore). Tryptic peptides were analyzed using an LTQ Orbitrap Velos mass 201 spectrometer (Thermo Fisher Scientific) and the resulting MS/MS data were searched using 202 SEAQUEST on DISCOVERER (Thermo Fisher Scientific, v 1.3.0.339) against masses of 203 theoretical fragments from a database that included our translated transcriptome sequences, and 204 all chelicerate sequences in NCBI (downloaded 4/23/2015), totaling 171,068 sequences. Matches 
205 required a fragment ion mass tolerance of $0.80 \mathrm{Da}$ and a parent ion tolerance of $10.0 \mathrm{ppm}$;

206 oxidation of methionine and carbamidomethyl of cysteine were specified in Seaquest as variable

207 modifications. The Seaquest output was organized in Scaffold (Proteome Software Inc, v 4.4.3).

208 Peptides were identified with $90 \%$ minimum threshold and $0 \%$ false discovery rate (FDR) and 209 proteins were identified with $100 \%$ minimum threshold and 0\% FDR.

211 RESULTS

212 Data archiving All raw read data are available through the NCBI short read archive (SRA 213 accession number SRP118124). Results and data files associated with proteomics

214 (https://dx.doi.org/10.6084/m9.figshare.5378308;

215 https://dx.doi.org/10.6084/m9.figshare.5378299), gene family clustering

216 (https://dx.doi.org/10.6084/m9.figshare.5420986), gene ontology/annotations

217 (https://dx.doi.org/10.6084/m9.figshare.5421655), and differential expression

218 (https://dx.doi.org/10.6084/m9.figshare.5421517) are available via Figshare.

219 Transcriptome The final trimmed read files comprised 43,550,457 + $(20,370,960$ left; 20,370,960 220 right; 2,808,555 merged), 48,457,662 Оे (22,783,475 left; 22,783,475 right; 2,890,675 merged), 221 and 92,008,100 combined (male and female reads added together) reads. The Trinity assemblies 222 produced $16,799+(\mathrm{N} 50=745 ; 9,904,215$ total bases $), 24,351$ ○े $(\mathrm{N} 50=464 ; 10,664,050$ total 223 bases), and 38,021 combined ( $\mathrm{N} 50=661 ; 20,752,384$ total bases) contigs. BUSCO estimations of 224 completeness show the combined assembly out performs the sex specific assemblies in capturing 225 core single copy orthologs (\% missing: 87 +; 91 万ै; 79 combined). These completeness results 226 likely have such high percentages of missing core orthologs due to sequencing material from such 227 highly specific venom gland tissues. Unless otherwise noted, the combined transcriptome was used 
228 in the remainder of analyses. ORF predictions produced 23,624 putative peptides, and functional

229 annotations were obtained for 8,075 out of 28,241 Trinity identified "genes" in the combined

230 assembly, not to be confused with contigs that include isoforms and alleles of individual genes.

231 A flow chart summary of steps we used to identify the set of transcripts ("entities") that are

232 venom-expressed (detected in the proteome) and dimorphic is presented in Figure 1B. We use

233 "entities" to refer to the distinct unique sets of venom components and define entities as the total

234 number of clusters (homologous groups) and singleton sequences. MCL analysis identified 10,581

235 entities in the combined transcripts. Of these $87 \%$ are dimorphic - 53\% are found only in males

236 and $47 \%$ in females. GFOLD analysis identified 3,800 (out of 23,634-16.07\%) differentially

237 expressed transcripts, indicating sexual dimorphisms in a wide range of gene including, but not

238 limited to, venom cocktail peptides/proteins (1,430 female upregulated; 2,370 male upregulated).

239 Proteome Crude venom separations of male and female venoms show that the profiles of expressed

240 proteins are quite different (Figure 1C), evidence that is supported by proteomics. LC-MS/MS

241 produced 3,205 spectra that corresponded to 62 distinct proteins in 31 clusters, which correspond

242 to 31 entities (Figure 1B), only $0.29 \%$ of total entities. Of these, nine are male-specific, non-

243 metabolic proteins, at least eight of which have no significant homology $\left(\mathrm{e} \leq 10^{-5}\right)$ with any

244 sequences in databases searched including NCBI and Arachnoserver (Figure 1C, Table 1A).

245 The results below detail the entities that were dimorphic and confirmed as venom

246 components through detection in proteomes.

247 Cluster 6 The most abundant, dimorphic cluster, the sixth most highly represented in the

248 combined transcriptome (66 homologous proteins, 38,922 mapped reads), included the proteins

249 with the highest number of distinct proteins (12) and represent $30.7 \%$ of MS spectra in the male

250 proteome (Table 1). Transcripts in this cluster appear to code for proteins of sizes consistent with 
251 the large proteins unique to male venoms (Fig 1C). While none of the assembled transcripts are

252 full length (initiating methionine through stop codon) individual transcripts in this cluster translate

253 into proteins ranging from 41-1093 aa. Multiple sequence alignment of these proteins generates a

254 consensus sequence of 1158 aa in length, and the longest single transcript in the alignment (1093

255 aa) has a predicted MW of $128.95 \mathrm{kDa}$. The homologs in this cluster are grouped by MCL into

256 eight sub-clusters and five singletons (Figure 2).

257 Proteins in cluster 6 contain multiple repeating units, each of which has $\sim 150$ aa and a

258 conserved pattern of 10 cysteine residues (Figure 3). Submission of a multiple alignment of these

259 repeats to the Fold and Function Assignment (FFAS) server (http://ffas.sanfordburnham.org/ffas-

260 cgi/cgi/ffas.pl; PMID 15980471) returns strong evidence of distant homology to Argos, a 223-

261 residue antagonist of epidermal growth factor receptor signaling with a known structure (PDB ID

$2623 \mathrm{c} 9 \mathrm{a})$. Argos contains 3 small $\beta$-sheet rich domains, the first two of which correspond to one of

263 the sequence repeats present in the cluster 6 proteins, with a similar pattern of 10 cysteine residues

264 making five disulfide bonds (Figure 3B). The third Argos domain corresponds to an extra half-

265 repeat with six of the 10 cysteines and three disulfide bonds (Figure 3B). Argos uses contacts from

266 multiple domains to bind and sequester small protein ligands of EGF receptors. Based on the FFAS

267 score (-15), it is quite likely that the cluster 6 proteins have a similar fold, disulfide pattern, and

268 domain organization to Argos; however, the sequence homology to Argos is so distant $(<20 \%$

269 sequence identity between Argos domains 1 and 2 and any sequence repeat in

270 the Tetragnatha proteins as shown in Figure 3A) that a functional similarity is much less certain.

Other male-specific venom proteins Seven additional male-specific clusters include

272 transcripts that code for proteins that span 20-37 kDa. These are sizes that correspond to predicted

273 molecular weights of full-length proteins within each cluster (Fig 1C). Cluster numbers are labeled 
274 next to individual bands in Figure $1 \mathrm{C}$ and correspond to the relative rankings based on

275 representation in the combined transcriptome: cl-2448 (>36 kDa), cl-217 (37 kDa), cl-218 (35

$276 \mathrm{kDa}), \mathrm{cl}-1113(26 \mathrm{kDa})$, cl-9248 (>26 kDa), cl-7374 (>24 kDa), and cl-2111 (21 kDa). All were

277 detected in the proteome, though not as abundantly as cluster 6. Proteins encoded by genes in all

278 of these clusters correspond to novel gene families without homologous sequences present in

279 existing databases, including GenBank and Arachnoserver. Proteins in each of these clusters have 280 multiple cysteine residues (8-21), and with the exception of clusters 217 and 218, they have at least 281 two CxC motifs.

282 In addition to identifying novel families of proteins in these venoms, we also identified 283 several sequences with evidence of homology to known enzymes. Venom proteins in the male 284 venom hit three large peptidases: M13 metalloendopeptidase (specifically neprilysin, $\sim 90 \mathrm{kDa}$ ), 285 M14 carboxypeptidase M (sequence not full length, but estimated to be $>55 \mathrm{kDa}$ ), and S8 peptidase 286 (specifically neuroendocrine convertase, $\sim 72 \mathrm{kDa}$ ). Only one large peptidase - M13 287 metalloendopeptidase - was identified in the venom proteome of females; however, homologous 288 sequences to each were found in both transcriptomes. Both S8 and M14 peptidase family members 289 are known to be involved with activation and processing/regulation of hormones, respectively, 290 whereas M13 peptidases are comprised of GluZincins, a superfamily of peptidases that act on 291 molecules < 40 aa (MEROPS peptidase database, http://merops.sanger.ac.uk/index.shtml) and 292 have also been reported in venom of a trapdoor spider (Undheim et al., 2013). The degradative 293 enzymes hyaluronidase and phospholipase A2 (PLA2) were identified in the venom of females, 294 constituting $\sim 1 \%$ of the proteome. Cluster 19 contains peptides corresponding to various 295 hemocyanin subunits and represents the most abundant set of proteins detected in the female 296 venom proteome (Table 1B). Each subunit varies in size, and the most prominent was subunit G, 
297 which is predicted to be $\sim 72 \mathrm{kDa}$ (Figure 1C). The female venom is also rich in small cysteine-

298 rich peptides, corresponding to clusters 2335, 846, and 8293 identified in the proteome (Table 1A).

299 Within gene families observed only in male proteomes, we recovered interesting patterns

300 of sexually dimorphic expression and potential functions. Despite some mRNA from females

301 mapping to these transcripts, their peptides were not detected in the female proteome. This could

302 be due to a lack of translation following transcription or perhaps the proteins are not present in the 303 venom. GO enrichment analyses performed on all male-only peptides, based on the proteomics 304 analysis, indicated potential non-feeding functions of these proteins. Four GO terms were 305 significantly enriched, two relating to hormone functions - GO:0016486 (BP peptide hormone 306 processing; FDR p-value = 0.0143), GO:0008237 (MF metallopeptidase activity; FDR p-value = 307 0.0349), GO:0042445 (BP hormone metabolic process; FDR p-value= 0.0349), and GO:0006518 308 (BP peptide metabolic process; FDR p-value= 0.0497). Half of the enriched GO terms were 309 specifically associated with hormone functions, suggesting this venom-based sexual dimorphism 310 could be involved in sexual communication.

\section{DISCUSSION}

313 The results we present provide a first identification and characterization of unique and sexually 314 dimorphic components in venoms of Tetragnatha versicolor. Combined proteomics and 315 transcriptomics identify proteins that are expressed in venoms and confirm the presence of sexually 316 dimorphic expression of particular components. The majority of components we identify are 317 sufficiently different from proteins in databases to be able to annotate with confidence using 318 homology searching. Recovering a low annotation percentage and high protein-coding 319 compliment (in terms of genes and isoforms) are both consistent with previous genomic studies of 
320 spider taxa (Croucher et al., 2013; Sanggaard et al., 2014; Brewer et al., 2014) and illustrates the

321 early nature and promise of spider genome biology. Additionally, it is likely that tetragnathid

322 spiders will have many novel genes and gene families, as this family has not previously been the

323 subject of deep sequencing efforts.

324 Interestingly, this study shows that the majority of proteins identified in the T. versicolor

325 venom proteome are sexually dimorphic ( $\sim 87 \%)$, with 4,908 distinct proteome entities only in

326 adult males (Figure 1). Most of these had no detected corresponding transcripts in female venom

327 gland tissues and are not present in female venom cocktails. However, a small number of sequence

328 reads from females map to transcripts of male-only peptides, (and vice versa) indicating these may

329 be expressed in females but not translated or not incorporated into the female venom cocktail. Of

330 the "high molecular weight", male-only proteins in the venom proteome, only three of 23

331 corresponding transcripts are not differentially expressed between the sexes, as indicated by non-

332 significant GFOLD values. While the males have more unique components, there are 4,269 unique

333 female proteome entities, including small number of unique low molecular weight peptides.

334 The unique peptides in female venoms are homologous to other spider venom peptides,

335 range in size from 5.9-7.9 $\mathrm{kDa}$, and have ICK motifs (-C6C-CC-C-C-) that are consistent with

336 them functioning as neurotoxins involved in prey immobilization. The biased presence of

337 potentially toxic peptides in female venoms is consistent with observations of differences between

338 males and females in concentration of low molecular weight components (Binford, Gillespie \&

339 Maddison, 2016, Figure 1C). This pattern mirrors within sex, among species differences in

340 Hawaiian Tetragnatha that have evolved differences in feeding biology. Specifically, as part of an

341 adaptive radiation within Hawaiian Tetragnatha, a clade lost web-building behavior and evolved

342 to be wandering foragers with an associated shift in dietary niche. With that evolutionary transition 
343 to wandering the lineage underwent a coincident reduction in low molecular weight venom

344 peptides (Binford, 2001). Given that evolutionary shifts in low molecular weight peptides in

345 venoms appear to occur in association with shifts in feeding biology, the lack of detection of venom

346 peptides in males may be best explained by differences in adult niche that lead to a reduction in

347 male reliance on venom peptides for prey immobilization (Binford, Gillespie \& Maddison, 2016).

348 The more striking dimorphism that is less easily explainable by differences in dietary niche

349 is in the male specific novel proteins, the "cluster 6" proteins. These belong to a single gene family

350 with estimated molecular weights corresponding to proteins detected with 1-D protein gels across

351 a comparative sampling of Tetragnatha (Fig 1,(Binford, Gillespie \& Maddison, 2016). The

352 rationale for suggesting a possible role beyond feeding is that these "cluster 6" components

353 comprise a high proportion of the male-specific proteins (12 of 23 unique proteins; next largest

354 family 2 of 23; Table 1A), suggesting an important functional role unique to males. While BLAST

355 searches of these male-specific proteins did not detect homology to known sequences, predicted

356 structural homology to Argos proteins that bind ligands to epidermal growth factors suggests

357 potential for binding to small proteins. Moreover, functional annotations and GO enrichment

358 analyses suggest hormone-related functions. Due to the degree of similarity in motifs, and likely

359 homology, between Argos and the novel male-only "cluster 6" proteins discovered in T. versicolor,

360 we propose the name Argoinonui (Argo for the Argos protein, ino is Hawaiian for "venom", and

361 nui is Hawaiian for "large") for this high-molecular weight venom gene family.

362 The high molecular weight components in male T. versicolor venom may be pervasive in

363 the genus Tetragnatha, based on previous 1-D gel studies of venom peptide diversity (Binford,

364 Gillespie \& Maddison, 2016). One striking aspect of this genus of spiders is their very unusual

365 sexual behavior: While courtship in most spiders involves an elaborate and extended period of 
366 vibrational or visual communication, in most Tetragnatha there is little evidence for

367 communication prior to the male and female approaching each other. They connect physically by

368 spreading the chelicerae wide and locking fangs (Figure 1A), involving a dorsal spur on the male

369 chelicerae. The cheliceral-locking mechanism apparently precludes the need for epigynal coupling

370 and is associated with secondary loss of a sclerotized epigynum (Levi, 1981). These alterations of

371 mating morphologies combined with the presence of male-only components of the venom cocktail

372 that are likely not used in feeding or defense, lead to a hypothesis that the unique male components

373 in venom play some as yet undescribed role in mating biology (Binford, Gillespie \& Maddison,

374 2016).

375 While sexual differences in venom composition driven by adult niche are likely, venoms

376 are also known to play a role in sexual biology (Polis \& Sissom, 1990) and thus may be under the

377 influence of sexual selection. As secreted molecules with intra-individual functionality, they have

378 potential for biological roles in sex (Binford, Gillespie \& Maddison, 2016). The origin of the male-

379 specific proteins appears to have been coincidental with the origin of the unusual premating

380 cheliceral-locking behavior by which these spiders intertwine their fangs while mating (Fig 1a).

381 Members of the family Tetragnathidae are secondarily haplogyne, having lost much of the

382 complexity in male and female genitalia that often functions in maintaining species boundaries

383 and mate recognition, evolving via sexual selection by female choice. The presence of cheliceral

384 locking during mating in many tetragnathid species provides an alternative mechanism upon which

385 sexual selection and mate recognition may have evolved. This has been demonstrated in the

386 tetragnathid species Leucauge venusta where tactile stimulation of females is accomplished via

387 specialized male setation during cheliceral locking (Aisenberg, Barrantes \& Eberhard, 2015). 

components. First, the possibility that the two groups of high molecular weight proteins are not involved in feeding or defense is supported by the significant homology of these components to

391 hormone processing peptidases (BLAST e-value $\sim 0.0$ ). If true, then we would expect to find these 392 high molecular weight proteins in all spiders that show cheliceral-locking behavior, but not in 393 those without; while preliminary data for a small number of Hawaiian and mainland Tetragnatha 394 support this hypothesis (Binford, Gillespie \& Maddison, 2016), clearly more data are needed. 395 Second, if the high molecular weight components in the venom are playing a sexual function, then 396 we might expect that recognition could be compromised at some level in taxa that display 397 cheliceral-locking behavior but do not have high molecular weight proteins. Here again, an 398 intriguing observation in support of this argument is the finding of prolonged male-to-male 399 cheliceral locking and mating attempts in the tetragnathid spider Doryonychus raptor (Gillespie, 400 1991), a species which lacks dimorphic venom(Binford, Gillespie \& Maddison, 2016), suggesting 401 that recognition is less complete than in most other taxa that employ this mating strategy and 402 exhibit dimorphic venoms.

403 While it is interesting that the cheliceral-locking mating behavior and dimporphic venoms 404 coincide, especially considering mate recognition is only known to be complete in species 405 possessing both, several caveats exist. The male-only peptides present in $T$. versicolor are large $406(>100 \mathrm{kDa})$, unlike known volatile pheromones (Wyatt, 2014a) and a mechanism for their transfer 407 to females is presently unknown. Additionally, a previous study of spider venom sexual 408 dimorphisms in the distantly related and non-cheliceral locking species Phoneutria nigriventer 409 (Araneae: Ctenidae) indicated male-only, high molecular weight components (Herzig, Ward \& 410 Santos, 2002) a pattern similar to T. versicolor. No known mating mechanism has been ascribed 
411 to the $P$. nigriventer dimorphism. Much like Hawaiian Tetragnatha, both sexes of P. nigriventer

412 wander in search of prey, but we do not know whether these genes are homologous and cannot

413 speculate on any shared function.

414 Regardless of the functions, a striking sexual dimorphism is present in all species of 415 Tetragnatha examined to date. Our results provide the first sequence-level investigation of the 416 family Tetragnathidae and highlight the diversity of spider-produced chemicals awaiting discovery 417 in understudied groups.

\section{CONCLUSIONS}

The genus Tetragnatha exhibits striking sexual dimorphisms in venom composition

421 (Binford, Gillespie \& Maddison, 2016) Herein, we have documented the specifics of this

422 dimorphism in the species $T$. versicolor using deep sequencing of transcriptomes and mass

423 spectrometric proteomic validation of transcript translation. While the results are still preliminary,

424 the venom of Tetragnatha spiders may function in both mate recognition as well as adaptive 425 specialization for prey, in which case venom could provide insights into the genomic 426 underpinnings of adaptive radiation as well as the interplay between plasticity and variability in 427 fostering species proliferation. The venom of $T$. versicolor includes proteins and peptides 428 comprising a wide array of molecular weights, as well as many novel compounds. Many of the 429 dimorphic components cannot be associated with any currently characterized peptides. Previous 430 work has demonstrated sexually dimorphic venoms can facilitate each mature sex occupying 431 different feeding niches, and several of the female-only components present in $T$. versicolor are 432 homologous to traditional feeding and defensive toxins. Males possess several unique gene 433 families, many of which cannot be annotated. A newly discovered high molecular weight and 
434 male-only gene family, deemed Argoinonui, is associated with gene ontologies relating to hormone

435 processing and regulation and has FFAS indicated homology to the Drosophila protein Argos, an

436 epidermal growth factor associated protein.

437

438

ACKNOWLEDGEMENTS

439

The authors would like to thank David and Diana Binford for help collecting on the farm; Linda

Breci and Cynthia David for proteomic work at the RDI Analytical/Biological Mass Spectrometry

441

Core, University of Arizona; and Chase Magsig, Aayushi Patel, and Augustus Floyd for help

442

collecting North Carolina Spiders.

443

444

445

446

447

448

449

450

451

452

453

454

455

456

457

458

459

460

461

462

463

464

465

466

467

468

469

\section{REFERENCES}

Aisenberg A, Barrantes G, Eberhard WG 2015. Hairy kisses: tactile cheliceral courtship affects female mating decisions in Leucauge mariana (Araneae, Tetragnathidae). Behavioral ecology and Sociobiology, 69(2), 313-323. DOI: 10.1007/s00265-014-1844-2.

Bateman A, Coin L, Durbin R, Finn RD, Hollich V, Griffiths Jones S, Khanna A, Marshall M, Moxon S, Sonnhammer EL 2004. The Pfam protein families database. Nucleic Acids Research 32:D138-D141.

Berns CM 2013. The evolution of sexual dimorphism: understanding mechanisms of sexual shape differences. In: Moriyama H ed. Sexual Dimorphism. Sexual Dimorphism. Rijeka: InTech, Ch. 01. DOI: 10.5772/55154.

Binford GJ 2001. Differences in venom composition between orb-weaving and wandering Hawaiian Tetragnatha (Araneae). 74:581-595. DOI: 10.1006/bij1.2001.0592.

Binford GJ, Gillespie RG, Maddison WP 2016. Sexual dimorphism in venom chemistry in Tetragnatha spiders is not easily explained by adult niche differences. Toxicon 114:45-52. DOI: 10.1016/j.toxicon.2016.02.015.

Blackledge TA, Gillespie RG 2004. Convergent evolution of behavior in an adaptive radiation of Hawaiian web-building spiders. 101:16228-16233. DOI: 10.1073/pnas.0407395101.

Bolger AM, Lohse M, Usadel B 2014. Trimmomatic: a flexible trimmer for Illumina sequence data. Bioinformatics 30:2114-2120. DOI: 10.1093/bioinformatics/btu170.

Brewer MS, Carter RA, Croucher PJP, Gillespie RG 2014. Shifting habitats, morphology, and selective pressures: Developmental polyphenism in an adaptive radiation of Hawaiian spiders. Evolution 69:162-178. DOI: 10.1111/evo.12563.

Croucher PJP, Brewer MS, Winchell CJ, Oxford GS, Gillespie RG 2013. De novo characterization of the gene-rich transcriptomes of two color-polymorphic spiders, Theridion grallator and T. californicum (Araneae: Theridiidae), with special reference to pigment genes. Bmc Genomics 14:862. DOI: 10.1186/1471-2164-14-862. 
470 D'Suze G, Sandoval M, Sevcik C 2015. Characterizing Tityus discrepans scorpion venom from a

471

472

473

474

475

476

477

478

479

480

481

482

483

484

485

486

487

488

489

490

491

492

493

494

495

496

497

498

499

500

501

502

503

504

505

506

507

508

509

510

511

512

513

514

515 fractal perspective: Venom complexity, effects of captivity, sexual dimorphism, differences among species. Toxicon 108:62-72. DOI: 10.1016/j.toxicon.2015.09.034.

Dobin A, Davis CA, Schlesinger F, Drenkow J, Zaleski C, Jha S, Batut P, Chaisson M, Gingeras TR 2012. STAR: ultrafast universal RNA-seq aligner. Bioinformatics 29:15-21. DOI: 10.1093/bioinformatics/bts635.

Elias DO, Mason AC 2014. The role of wave and substrate heterogeneity in vibratory communication: practical issues in studying the effect of vibratory environments in communication. Studying vibrational communication. DOI: 10.1007/978-3-662-43607-3_12.

Enright AJ, Van Dongen S, Ouzounis CA 2002. An efficient algorithm for large-scale detection of protein families. Nucleic Acids Research 30:1575-1584.

Escoubas P 2006. Molecular diversification in spider venoms: A web of combinatorial peptide libraries. Molecular Diversity 10:545-554. DOI: 10.1007/s11030-006-9050-4.

Feng J, Meyer CA, Wang Q, Liu JS, Shirley Liu X, Zhang Y 2012. GFOLD: a generalized fold change for ranking differentially expressed genes from RNA-seq data. Bioinformatics 28:2782-2788. DOI: 10.1093/bioinformatics/bts515.

Finn RD, Clements J, Eddy SR 2011. HMMER web server: interactive sequence similarity searching. Nucleic Acids Research:gkr367.

Fry BG, Roelants K, Champagne DE, Scheib H, Tyndall JDA, King GF, Nevalainen TJ, Norman JA, Lewis RJ, Norton RS, Renjifo C, la Vega de RCR 2009. The toxicogenomic multiverse: convergent recruitment of proteins into animal venoms. Annual Review of Genomics and Human Genetics 10:483-511. DOI: 10.1146/annurev.genom.9.081307.164356.

Gillespie RG 1987. The mechanism of habitat selection in the long-jawed orb-weaving spider Tetragnatha elongata (Araneae, Tetragnathidae). 15:81-90. DOI: 10.2307/3705511?ref=noX-route:542c24af2497b197f3b3f4b547bf353c.

Gillespie RG 1991. Hawaiian spiders of the genus Tetragnatha: I. Spiny Leg Clade. 19:174-209. DOI: 10.2307/3705891?ref=no-x-route:906800179fef60d97f0980df3a26b642.

Gillespie RG 2004. Community assembly through adaptive radiation in Hawaiian spiders. 303:356-359. DOI: 10.1126/science.1091875.

Gillespie RG 2005. Evolution of Hawaiian spider communities. American Scientist 93:122-131. Gray MR, Sutherland SK 1978. Venoms of Dipluridae. In: Arthropod venoms. Springer, 121148.

Haas BJ, Papanicolaou A, Yassour M, Grabherr M, Blood PD, Bowden J, Couger MB, Eccles D, Li B, Lieber M, Macmanes MD, Ott M, Orvis J, Pochet N, Strozzi F, Weeks N, Westerman R, William T, Dewey CN, Henschel R, Leduc RD, Friedman N, Regev A 2013. De novo transcript sequence reconstruction from RNA-seq using the Trinity platform for reference generation and analysis. Nature Protocols 8:1494-1512. DOI: 10.1038/nprot.2013.084.

Herzig V, Khalife AA, Chong Y, Isbister GK, Currie BJ, Churchill TB, Horner S, Escoubas P, Nicholson GM, Hodgson WC 2008. Intersexual variations in Northern (Missulena pruinosa) and Eastern (M. bradleyi) mouse spider venom. Toxicon 51:1167-1177. DOI: 10.1016/j.toxicon.2008.02.001.

Herzig V, Ward RJ, Santos dos WF 2002. Intersexual variations in the venom of the Brazilian “armed”spider Phoneutria nigriventer (Keyserling, 1891). Toxicon 40:1399-1406.

Herzig, V., Wood, D.L., Newell, F., Chaumeil, P.A., Kaas, Q., Binford, G.J., Nicholson, G.M., Gorse, D. and King, G.F., 2010. ArachnoServer 2.0, an updated online resource for spider toxin sequences and structures. Nucleic acids research, 39(suppl_1), pp.D653-D657. 
516 Isbister GK, Gray MR 2004. Bites by Australian mygalomorph spiders (Araneae,
$517 \quad$ Mygalomorphae), including funnel-web spiders (Atracinae) and mouse spiders

517

518

519

520

521

522

523

524

525

526

527

528

529

530

531

532

533

534

535

536

537

538

539

540

541

542

543

544

545

546

547

548

549

550

551

552

553

554

555

556

557

558

559

560

561

(Actinopodidae: Missulena spp). Toxicon 43:133-140. DOI: 10.1016/j.toxicon.2003.11.009.

King G 2015. Venoms to drugs: venom as a source for the development of human therapeutics. Royal Society of Chemistry.

Krogh A, Larsson B, Heijne von G, Sonnhammer ELL 2001. Predicting transmembrane protein topology with a hidden markov model: application to complete genomes. Edited by F. Cohen. Journal of molecular biology 305:567-580. DOI: 10.1006/jmbi.2000.4315.

Lagesen K, Hallin P, Rødland EA, Stærfeldt H-H, Rognes T, Ussery DW 2007. RNAmmer: consistent and rapid annotation of ribosomal RNA genes. Nucleic Acids Research 35:31003108 .

Langmead B, Salzberg SL 2012. Fast gapped-read alignment with Bowtie 2. Nature Methods 9:357-359. DOI: 10.1038/nmeth.1923.

Langmead B, Trapnell C, Pop M, Salzberg SL 2009. Ultrafast and memory-efficient alignment of short DNA sequences to the human genome. Genome biology 10:R25. DOI: 10.1186/gb2009-10-3-r25.

Levi HW 1981. American orb-weaver genera Dolichognatha and Tetragnatha north of Mexico (Araneae: Araneidae, Tetragnathinae).

Magoč T, Salzberg SL 2011. FLASH: fast length adjustment of short reads to improve genome assemblies. Bioinformatics 27:2957-2963. DOI: 10.1093/bioinformatics/btr507.

Maretić Z, Levi HW, Levi LR 1964. The theridiid spider Steatoda paykulliana, poisonous to mammals. Toxicon 2:149IN1151-150IN2154.

Martin M 2011. Cutadapt removes adapter sequences from high-throughput sequencing reads. EMBnet journal 17:pp-10.

Menezes MC, Furtado MF, Travaglia-Cardoso SR, Camargo ACM, Serrano SMT 2006. Sexbased individual variation of snake venom proteome among eighteen Bothrops jararaca siblings. Toxicon 47:304-312. DOI: 10.1016/j.toxicon.2005.11.007.

Miller DW, Jones AD, Goldston JS, Rowe MP, Rowe AH 2016. Sex differences in defensive behavior and venom of the striped bark scorpion Centruroides vittatus (Scorpiones: Buthidae). Integrative and Comparative Biology 56:1022-1031. DOI: 10.1093/icb/icw098.

National Center for Biotechnology Information (NCBI)[Internet] 1988. Bethesda (MD): National Library of Medicine (US), National Center for Biotechnology Information - [cited 2015 Apr 23]. Available from: https://www.ncbi.nlm.nih.gov/

Polis GA, Sissom WD 1990. Life history. The biology of scorpions:161-223.

Quinlan AR, Hall IM 2010. BEDTools: a flexible suite of utilities for comparing genomic features. Bioinformatics 26:841-842. DOI: 10.1093/bioinformatics/btq033.

Rash LD, King RG, Hodgson WC 2000. Sex differences in the pharmacological activity of venom from the white-tailed spider (Lampona cylindrata). Toxicon 38:1111-1127.

Sanggaard KW, Bechsgaard JS, Fang X, Duan J, Dyrlund TF, Gupta V, Jiang X, Cheng L, Fan D, Feng Y, Han L, Huang Z, Wu Z, Liao L, Settepani V, gersen IBTO, Vanthournout B, Wang T, Zhu Y, Funch P, Enghild JJ, Schauser L, Andersen SU, Villesen P, Schierup MH, Bilde T, Wang J 2014. Spider genomes provide insight into composition and evolution of venom and silk. Nature Communications 5:1-11. DOI: 10.1038/ncomms4765.

Shannon P, Markiel A, Ozier O, Baliga NS, Wang JT, Ramage D, Amin N, Schwikowski B, Ideker T 2003. Cytoscape: a software environment for integrated models of biomolecular interaction networks. Genome Research 13:2498-2504. 
562 Simão FA, Waterhouse RM, Ioannidis P, Kriventseva EV, Zdobnov EM 2015. BUSCO:

563 assessing genome assembly and annotation completeness with single-copy orthologs.

564 Bioinformatics 31:3210-3212. DOI: 10.1093/bioinformatics/btv351.

565 World Spider Catalog 2018. World Spider Catalog. online at http://wsc.nmbe.ch, version 19.0.

566 Natural History Museum Bern.

567 Wyatt TD 2014a. Proteins and peptides as pheromone signals and chemical signatures. Animal 568 Behaviour 97:273-280.

569 Wyatt TD 2014b. Pheromones and Animal Behavior. Cambridge University Press.

570 Young MD, Wakefield MJ, Smyth GK, Oshlack A 2010. Gene ontology analysis for RNA-seq: 571 accounting for selection bias. Genome biology 11:R14. 


\section{Figure 1}

Comparative analysis of Tetragnatha male and female venom gland transcriptomes

A) Diagram shows cheliceral locking between male and female spiders during mating. B)

Flowchart shows the pipeline of transcriptome clustering analysis yielding sexually dimorphic entities detected in both transcriptomes and proteomes. C) SDS-PAGE (12\%) of $T$. versicolor crude venom from male (M) and female (F) spiders; proteins that correspond to identified clusters are labeled with "cl-" followed by the cluster number and assigned molecular weight sizes are based on predictions made from full-length amino acid sequences using the compute pl/MW tool (http://web.expasy.org/compute_pi/).

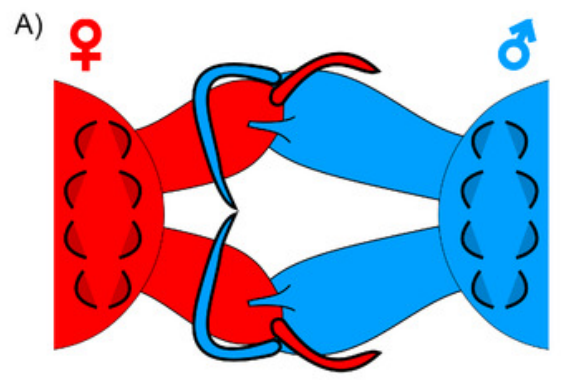

B)

total \#cDNA transcripts 38,021
2,963 clusters 7,618 singletons 10,581 entities

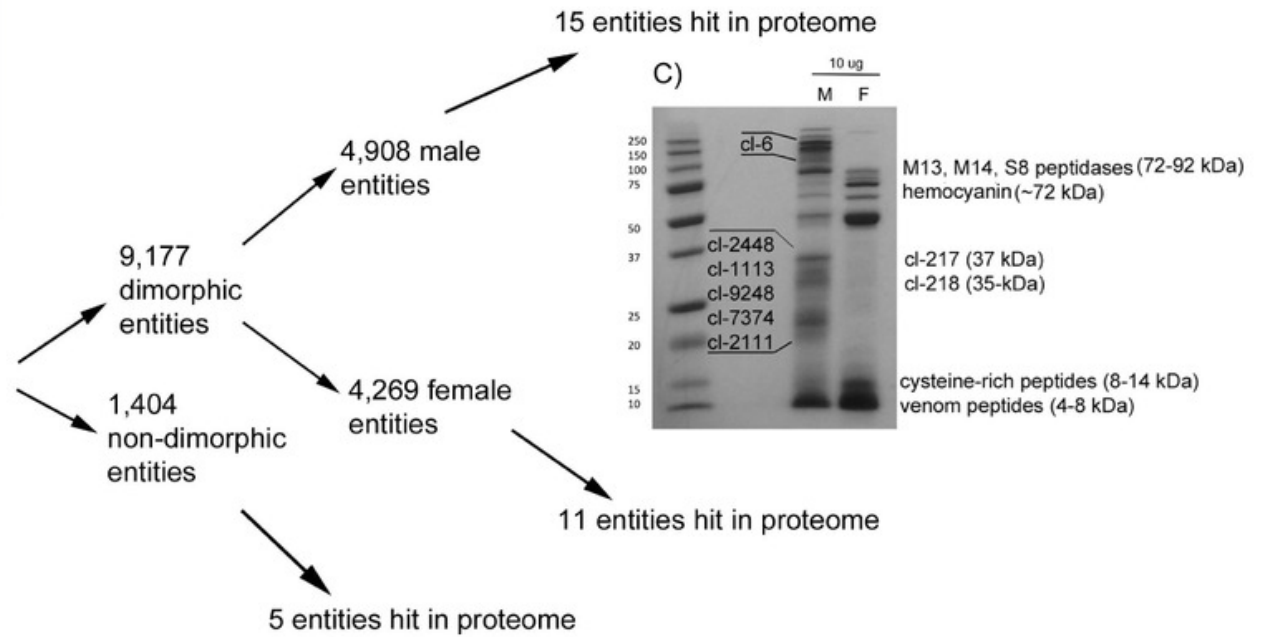




\section{Figure 2}

High molecular weight, male-only "gene family" and subclustering results from MCL analysis

A) Similarities between components of the high-molecular weight family of male-specific components, and B) subclustering of the same family. All members are present in both networks. These components show no similarities to known venom or toxin genes, bug gene ontology (GO) enrichment tests indicate a role in hormone signaling/transport. 


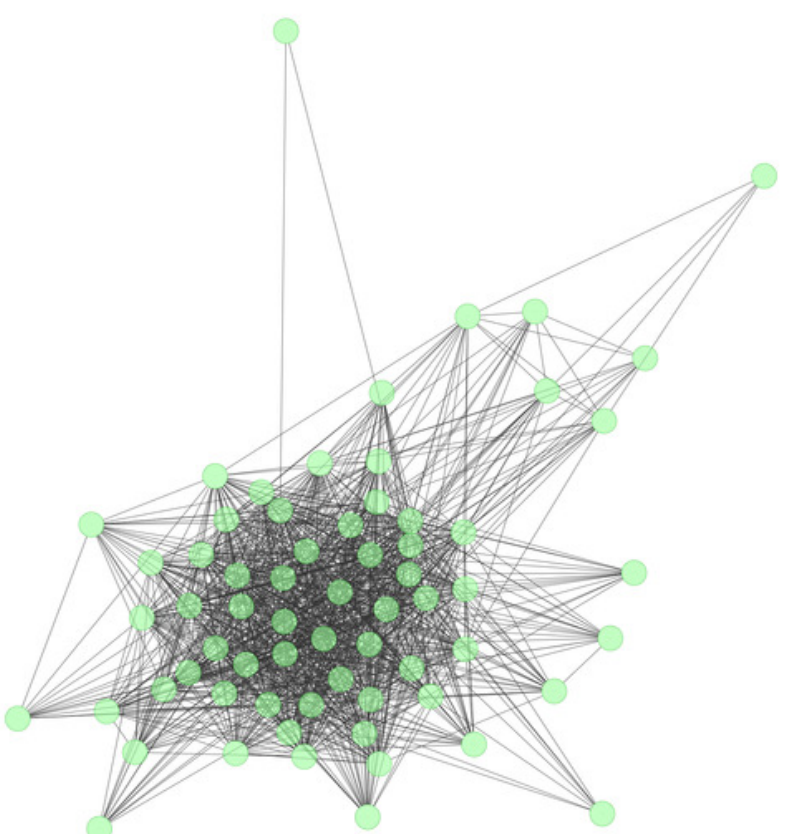

A

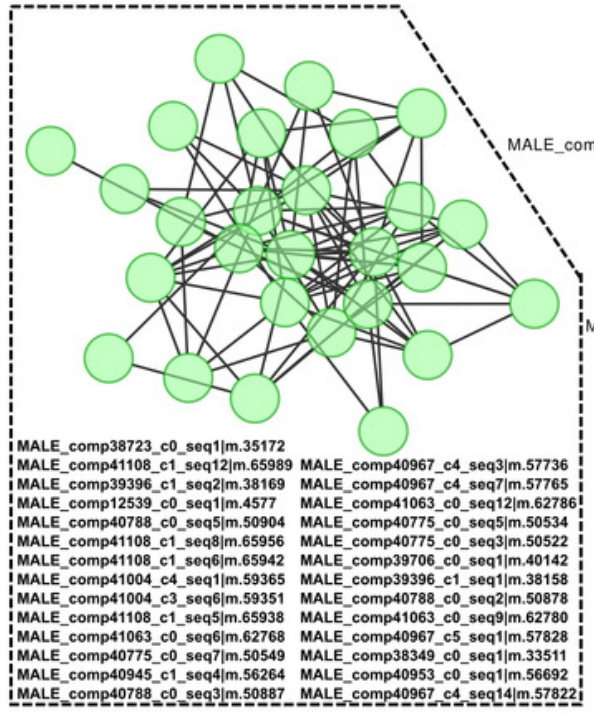

MALE_comp40788_co-seq3im.50887_MALE_comp40967.c4 seq14lm.57822i

MALE_comp41004_c3 seq7|m.59362

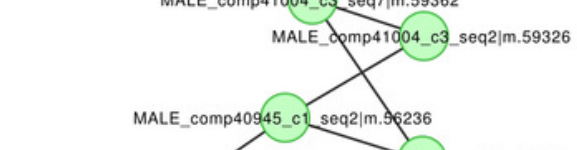

MALE_demp41004_c3_seq2|m.59326

6

MALE_comp40775_co-60.9/m.50541

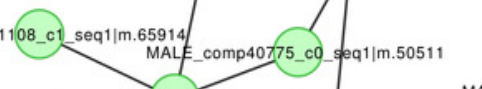

MALE_comp40967_c4 seg13|m.57818

MALE_comp40123_co_seq1/m.42694

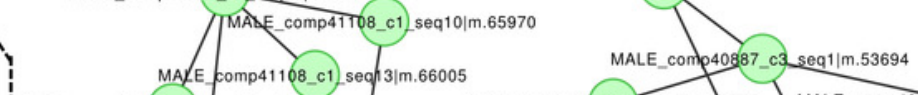

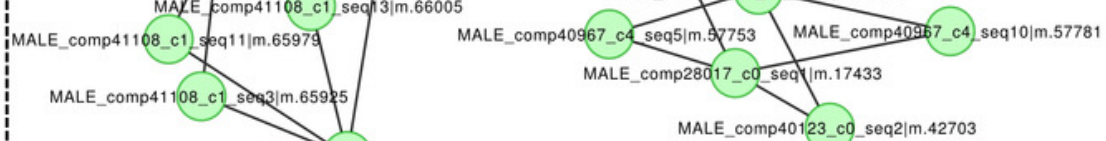

MALE_comp41004_c3_seq3/m.59331

MALE_comp40967_c4_seq4|m.57745

MALE_comp40775_co_seq4 4 m. 50527 MALE_comp40945_c1 seq 1 |m.56233

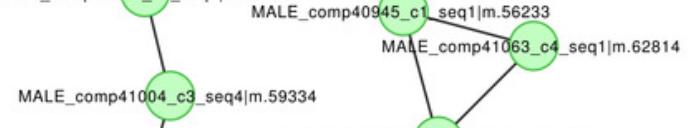

MALE_comp40953_c1_seq3|m.56702
MALE_comp40788_co_seq1|m.50874

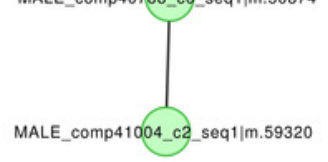

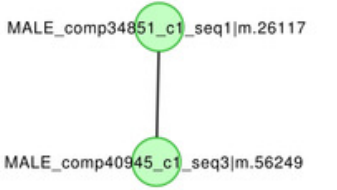

MALE_comp40967_c4_seq11|m.57791

MALE_comp41004_c3_seq5|m.59342

MALE_comp40967_cd_seq9|m.57771
MALE_comp41108_c1_seq9|m.65962

MALE_comp41016_c3_seq1|m.59857 
Figure 3

Remote homology of cluster 6 proteins to Drosophila Argos

A) Sequence alignments of Argos to each 6 repeats within a single cluster 6 protein from Tetragnatha; at top is the N-terminal half of each repeat aligned to domains 1 and 3 of Argos, while at bottom is the C-terminal half of each repeat aligned to domain 2 of Argos, B) Ribbon diagram of Argos structure (PDB ID 3c9a), colored by domain with disulfide bonds shown.

A

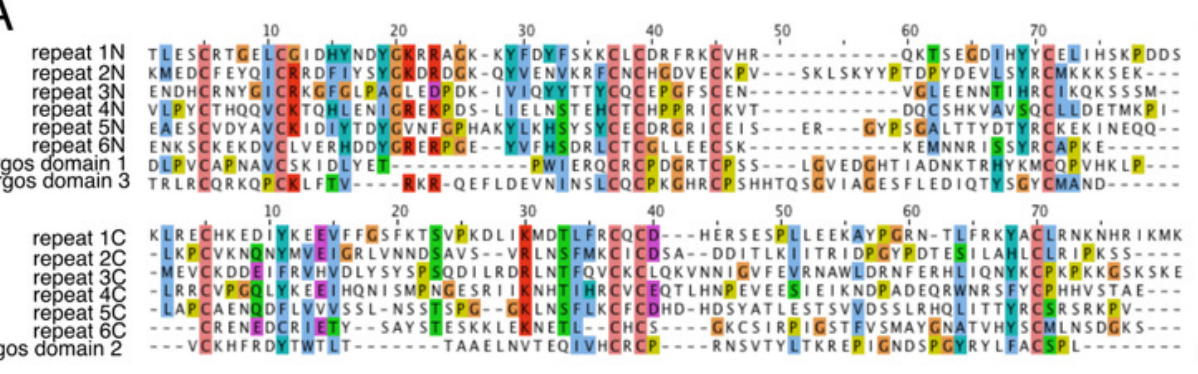

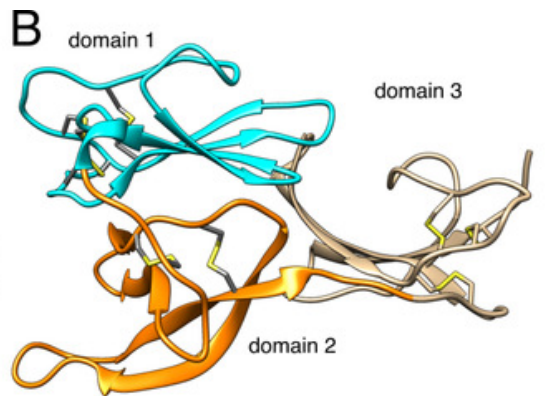




\section{Table $\mathbf{1}$ (on next page)}

Sexually dimorphic components of $T$. versicolor venom

Proteomics results are separated into four categories based on general function prediction hits from homology searches: unknown function, potentially toxic/defense proteins, potentially toxic peptides are presented in Panel A, and housekeeping/metabolism proteins are in panel B. Black boxes indicate an absence of a protein in the secreted venom. The symbol $(\bullet)$ indicates $<1 \%$ of total \#spectra. The total number of homologous polypeptides in each cluster is listed, along with the number of corresponding transcripts in the transcriptome. The symbol $\left(\wedge^{\wedge}\right)$ indicates a hit in the tryptic peptide search against the NCBI database. 
1 A.

\begin{tabular}{|c|c|c|c|c|c|c|c|}
\hline \multirow{2}{*}{$\begin{array}{l}\text { general function prediction based on } \\
\text { homology searches [cluster\#] }\end{array}$} & \multicolumn{2}{|c|}{$\begin{array}{l}\text { \#distinct } \\
\text { polypeptides } \\
\text { detected in } \\
\text { venom }\end{array}$} & \multicolumn{2}{|c|}{$\begin{array}{l}\text { \#spectra detected in } \\
\text { proteome (\% total) }\end{array}$} & \multicolumn{2}{|c|}{$\begin{array}{c}\text { \# homologous } \\
\text { polypeptides in } \\
\text { transcriptome cluster } \\
{[\mathrm{e}<10-5] \text { (\#transcripts) }}\end{array}$} & \multirow[t]{2}{*}{$\begin{array}{c}\text { top hit species in homology } \\
\text { searches (e-value) }\end{array}$} \\
\hline & $\mathbf{m}$ & $f$ & $\mathbf{m}$ & $\mathbf{f}$ & $\mathbf{m}$ & $\mathbf{f}$ & \\
\hline \multicolumn{8}{|l|}{ unknown function } \\
\hline new family (high MW) [6] & 12 & & $398(30.7)$ & & $66(38922)$ & $0(35)$ & - \\
\hline new family [1113] & 2 & & $25(1.9)$ & & $3(2639)$ & & - \\
\hline new family [9248] & 1 & & $10(*)$ & & $1(229)$ & & - \\
\hline new family [7374] & 1 & & $7(*)$ & & $1(661)$ & & - \\
\hline new family [2111] & 1 & & $33(2.5)$ & & $2(1382)$ & & - \\
\hline new family [2448] & 1 & & $8(*)$ & & $2(453)$ & & - \\
\hline new family [217] & 1 & & $16(1.2)$ & & $7(6492)$ & $1(195)$ & Cluex (e10-5) \\
\hline new family [218] & 1 & & $28(2.2)$ & & $8(15274)$ & $2(3)$ & - \\
\hline S8 peptidase [889] & 1 & & $14(1.1)$ & & $1(1107)$ & $3(886)$ & Stegodyphus (e0.0) \\
\hline M14 carboxypeptidase M [116] & 1 & 1 & $6(*)$ & & $7(751)$ & $6(766)$ & Stegodyphus (e0.0) \\
\hline M13 metalloendopeptidase [11] & 5 & 1 & $501(38.7)$ & $80(4.2)$ & $48(19836)$ & $13(11358)$ & Stegodyphus (e0.0) \\
\hline \multicolumn{8}{|l|}{ potentially toxic/defense proteins } \\
\hline chitinase [337] & 1 & 1 & $77(5.9)$ & $19(10.1)$ & $6(5557)$ & $1(2974)$ & Araneus (e0.0) \\
\hline venom allergen 5 [843] & 1 & 1 & $34(2.6)$ & $157(8.2)$ & $2(5401)$ & $2(86981)$ & Stegodyphus (e10-99) \\
\hline hyaluronidase [10277] & & 1 & & $6(*)$ & $0(62)$ & $1(690)$ & Brachypelma (e10-97) \\
\hline phospholipase A2 (PLA2) [10291] & & 1 & & $20(1.0)$ & $1(95)$ & $0(73)$ & Stegodyphus (e10-52) \\
\hline \multicolumn{8}{|l|}{ potentially toxic peptides } \\
\hline venom peptide [2335] & & 1 & & $22(1.2)$ & & $2(2043)$ & Nephila BLTX631 (e10-135) \\
\hline venom peptide [846] & & 2 & & $270(14.1)$ & 1 (139) & $3(43266)$ & Nephila BLTX631 (e10-24) \\
\hline venom peptide [8293] & & 1 & & $187(9.8)$ & $0(40)$ & $1(13297)$ & - \\
\hline
\end{tabular}


B.

7

\begin{tabular}{|c|c|c|c|c|c|c|c|}
\hline \multirow{2}{*}{$\begin{array}{l}\text { general function prediction based on } \\
\text { homology searches [cluster\#] }\end{array}$} & \multicolumn{2}{|c|}{$\begin{array}{l}\text { \#distinct } \\
\text { polypeptides } \\
\text { detected in } \\
\text { venom }\end{array}$} & \multicolumn{2}{|c|}{$\begin{array}{l}\text { \#spectra detected in } \\
\text { proteome (\% total) }\end{array}$} & \multicolumn{2}{|c|}{$\begin{array}{c}\text { \# homologous } \\
\text { polypeptides in } \\
\text { transcriptome cluster } \\
{[\mathrm{e}<10-5] \text { (\#transcripts) }}\end{array}$} & \multirow[t]{2}{*}{$\begin{array}{c}\text { top hit species in homology } \\
\text { searches (e-value) }\end{array}$} \\
\hline & m & f & m & $\mathbf{f}$ & $\mathbf{m}$ & $\mathbf{f}$ & \\
\hline \multicolumn{8}{|l|}{ housekeeping/metabolism proteins } \\
\hline hemocyanin (subunits $A, B, C, D, G$ ) [19] & & 10 & & $835(43.7)$ & $24(6550)$ & 14 (16316) & Stegodyphus (e0.0) \\
\hline hemocyanin (subunit D) & & 1 & & $32(1.7)$ & $\mathrm{n} / \mathrm{a}$ & $\mathrm{n} / \mathrm{a}$ & Latrodectus^ \\
\hline alpha amylase [4700] & & 1 & & $20(1.0)$ & $0(41)$ & $1(1777)$ & Stegodyphus (e10-144) \\
\hline alpha amylase [10595] & & 1 & & $37(1.9)$ & $0(2)$ & $1(514)$ & Lithobius (e10-98) \\
\hline alpha-2 macroglobulin [453] & 1 & & $6(*)$ & & $3(1006)$ & $4(997)$ & Hasarius (e0.0) \\
\hline acetylcholinesterase [831] & 1 & & $15(1.1)$ & & $2(9789)$ & $1(27)$ & Pardosa (e10-159) \\
\hline triacylglycerol lipase [75] & & 1 & & $33(1.7)$ & $9(1636)$ & $6(14009)$ & Stegodyphus (e10-159) \\
\hline protein tyrosine phosphatase rec. [231] & 1 & 1 & $19(1.5)$ & $12(*)$ & $3(5714)$ & $5(3671)$ & Homo (e10-70) \\
\hline corticotropin releasing factor [4347] & 1 & & $10(*)$ & & $1(138)$ & $1(1002)$ & Tribolium (e10-82) \\
\hline G-protein coupled rec. (GPRmth5) [117] & 1 & & $76(5.9)$ & & $10(2294)$ & $1(3)$ & Pediculus (e10-28) \\
\hline beta casein & 1 & & $6(*)$ & & $\mathrm{n} / \mathrm{a}$ & $\mathrm{n} / \mathrm{a}$ & Bos $^{\wedge}$ \\
\hline casein & 1 & & $6(*)$ & & $\mathrm{n} / \mathrm{a}$ & $\mathrm{n} / \mathrm{a}$ & Bos $^{\wedge}$ \\
\hline slit-like protein (leu-rich domain) [6912] & & 1 & & $7(*)$ & $0(2)$ & $1(100)$ & Stegodyphus (e10-20) \\
\hline
\end{tabular}

8 